\title{
Optimisation of masked ion irradiation damage profiles in YBCO thin films by Monte Carlo simulation
}

\author{
Nianhua Peng ${ }^{\mathrm{a}, *}$, Chris Jeynes ${ }^{\mathrm{a}}$, Roger Webb ${ }^{\mathrm{a}}$, Ivan Chakarov ${ }^{\mathrm{b}}$, \\ Mark Blamire ${ }^{\mathrm{c}}$ \\ a Surrey Centre for Research in Ion Beam Applications, School of Electronics, Computing and Mathematics, University of Surrey, \\ Guildford GU2 $7 X H, U K$ \\ b SILVACO International, Santa Clara, CA 95054, USA \\ ${ }^{\mathrm{C}}$ IRC in Superconductivity, University of Cambridge, Cambridge CB3 OHE, UK
}

\begin{abstract}
Systematic Monte Carlo simulation studies have been carried out to search for a possible optimal experimental condition on the irradiation damage profile production with a given mask structure. The results suggest that minimum ion scattering broadening tails could be achieved with projectile ranges in $\mathrm{Nb}$ mask about half of the mask thickness. Provided the projectile range to mask thickness is maintained, similar irradiation damage profiles could be created by different ions, including ions as heavy as $\mathrm{Cu}^{+}$.
\end{abstract}

(C) 2002 Elsevier Science B.V. All rights reserved.

Keywords: Monte Carlo simulation; Masked irradiation damage; Josephson junction

\section{Introduction}

Controlled formation of a small (up to $100 \mathrm{~nm}$ wide) nonsuperconducting barrier layer in between two superconducting electrodes is the central part of the $\mathrm{YBa}_{2} \mathrm{Cu}_{3} \mathrm{O}_{7-\delta}$ (YBCO) Josephson junction fabrication process. High quality YBCO Josephson junctions can be made using focused electron beam irradiation damage technique with beam energy up to a few hundred $\mathrm{keV}$, though the throughput is intrinsically low [1].

A combination of broad ion beam irradiation damage and high resolution mask structure ren-

\footnotetext{
${ }^{*}$ Corresponding author. Tel.: +44-1483-876093; fax: +441483-534139.

E-mail address: ees1np@eim.surrey.ac.uk (N. Peng).
}

ders opportunity for the mass production of YBCO Josephson junction at low cost and high yield. Experimental results reported so far include $\mathrm{O}^{+}, \mathrm{Ne}^{+}$and $\mathrm{H}^{+}$ion irradiation with various masks [2-4]. The preliminary experimental results are promising, but the device performances are not satisfactory in general, and differ from one group to another. The obvious reason for the poor performance is related to the poor definition of irradiation damage created barrier layers by different ion irradiation processes. Now a critical question appears: can junctions fabricated with different ions have the same damage profiles?

Direct observation of damage barrier layer is difficult, though it could be inferred from the performance of the device fabricated under different experimental conditions. An optimised fabrication 
condition is important for the successful manufacture of high quality devices. Considering the large amount of physical parameters relevant to the damage process, it is clear that the experimental optimisation process is definitely a time consuming and tedious one. Fortunately this optimisation process can be greatly simplified by using Monte Carlo simulation studies.

With a simple standard high resolution and high aspect ratio mask structure, here we report our recent Monte Carlo simulation results on the irradiation damage build-up with a series of ion beam species, for a brief quantitative estimation on the optimal experimental conditions for the Josephson junction fabrications using masked ion beam irradiation damage technique.

\section{Simulation code and simulation model}

The simulation code CRYSTAL was initially written to model the damage accumulation and channelling implantation in crystal silicon using the binary collision approximation [5]. It was adapted to include the orthorhombic crystal structure of YBCO, and had been employed successfully to study the sidewall scattering effect in high aspect ratio masked system [6] and beam condition dependent damage profile formations [7].

The two-dimensional simulation structure model is composed of a $400 \mathrm{~nm} \mathrm{Nb}$ mask with a fixed $50 \mathrm{~nm}$ opening, a $50 \mathrm{~nm}$ YBCO single crystalline target layer and a thick amorphous $\mathrm{LaAlO}_{3}$ substrate. The normal of the YBCO surface is (001) direction, and the mask opening wall is parallel to the $b c$ plane of YBCO crystal. The projection plane is chosen as the $a c$ plane of YBCO crystal and the implantation plane is defined by the ion beam direction and the normal of YBCO. The ion beam has been tilted by $15^{\circ}$ in accordance with the experimental condition, and the beam is aligned with the opening slot wall. No beam divergence is assumed in general.

\section{Simulation results and discussions}

It was anticipated before that a high energy beam has an advantage for Josephson junction fabrications for a restricted lateral spreading of ion beam scattering and uniform damage accumulation. This implies that the expected projectile range of energetic ions in an YBCO target should be greater than the thickness of an YBCO thin film by a reasonable factor, say 2 . The minimum ion energies required for a uniform irradiation damage in $50 \mathrm{~nm}$ YBCO thin films were derived from this criterion using SUSPRE code [8]. On the other hand, the maximum ion energy appropriate for masked ion beam irradiation process is limited by the effective stopping power of the $400 \mathrm{~nm} \mathrm{Nb}$ mask materials. For proton beam irradiation damage, ion energies in between 22 and $74 \mathrm{keV}$ should be able to meet the above general requirements. Similar energy windows can also be found out easily using SUSPRE code for other commonly available ions, as listed in Table 1 below.

Our previous simulation and experimental results revealed a substantial broadening of damage profiles compared to mask structures with $50 \mathrm{keV}$ proton beam irradiation process due to the enhanced mask sidewall scattering process [6]. This unwanted process is intrinsic for the small structures considered here, and can be reduced by using a thicker mask or a lower ion energy. To estimate the effect of sidewall scattering broadening on ion species, beam energies and mask structures quantitatively, here we introduce a ratio of mask thickness to projectile range in the mask, $r$, as a measure of the effective stopping power of mask structure to different energetic ions. The increase of mask stopping power by either reducing the projectile range in the mask or increasing the

Table 1

Estimated energy windows for different ion species with $400 \mathrm{~nm}$ $\mathrm{Nb}$ on $50 \mathrm{~nm}$ YBCO

\begin{tabular}{lcl}
\hline Projectile ion & $\begin{array}{l}\text { Minimum } \\
\text { energy }(\mathrm{keV})\end{array}$ & $\begin{array}{l}\text { Maximum } \\
\text { energy }(\mathrm{keV})\end{array}$ \\
\hline Proton & 22 & 74 \\
$\mathrm{He}$ & 44 & 98.7 \\
$\mathrm{O}$ & 135 & 341.2 \\
$\mathrm{Ne}$ & 160 & 352 \\
$\mathrm{Ar}$ & 275 & 655 \\
$\mathrm{Ni}$ & 390 & 863 \\
$\mathrm{Cu}$ & 405 & 902 \\
$\mathrm{Zn}$ & 415 & 936 \\
\hline
\end{tabular}




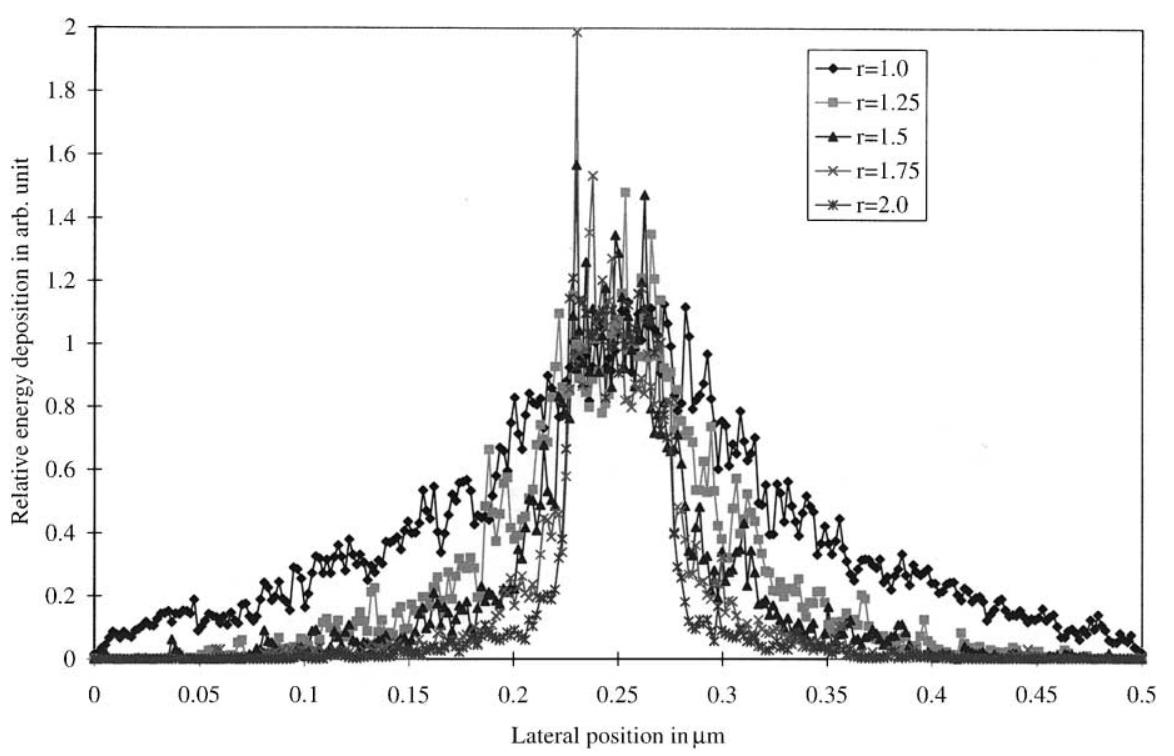

Fig. 1. Scaled proton irradiation damage distributions along the middle of YBCO thin film covered by a $400 \mathrm{~nm} \mathrm{Nb}$ mask with a 50 $\mathrm{nm}$ opening slot in the centre.

physical thickness can be treated equally in this way. For a standard $400 \mathrm{~nm} \mathrm{Nb}$ mask with $50 \mathrm{~nm}$ opening slot, the lateral distributions of proton beam irradiation damage accumulation in the middle of YBCO thin film are shown in Fig. 1, values of $r=1.0,1.25,1.50,1.75,2.0,2.5$ and 3.0 correspond to proton beam energies of $74.2,42.2$, 22.2, 20.4 and $16 \mathrm{keV}$ respectively. We scaled the damage levels by the average values in the central area defined by the mask structure for each individual irradiation process. It is evident that the tailing effect has been saturated for $r>2$.

A similar saturation of the broadening effect due to sidewall scattering has also been observed in the $\mathrm{O}^{+}$irradiation process. However, a smaller value of $r$ is needed for the same effect, indicating an interesting mass dependence in the ion irradiation process. This is because less sidewall scattering effect is expected from heavy ion irradiation. This can be demonstrated clearly by the simulation results of a series of masked irradiation damage profiles using different ions with the same $r$ value $(r=2)$. The scaled damage level distribution in the middle of the YBCO target is shown in Fig. 2. The similarity of damage profiles suggests that all ions have equivalent effects, independent of ion species, with $\mathrm{He}^{+}$irradiation as an exceptional case.
In our simulations only primary energy depositions are recorded, any further ion scattering cascades are ignored so far. It is possible that defect structure formed by heavy ion irradiation may be different from those formed with light ion irradiation, though the projectile ranges are similar. Furthermore, the application of heavy ion irradiation with a comparable $r$ factor means that the ion energy may go up to the $\mathrm{MeV}$ range, where the irradiation damage would create both point and extended defects. The production of extended defects is not useful for the barrier layer formation concerned here, since the superconductivity transition temperature is not very sensitive to the formation of extended defects [9]. Therefore, the best candidate ions for masked ion irradiation damage are the ions that encourage the creation of point defects, such as proton, $\mathrm{O}^{+}$and $\mathrm{Ne}^{+}$in the $\mathrm{keV}$ range. In addition to the production of lattice defects by irradiation damage process, reactive ion implant, say $\mathrm{Fe}$ implant in $\mathrm{YBCO}$, also has a dramatic effect in suppressing superconductivity, where the energy of ion beam is even lower than the minimum energy listed in the Table 1 [10]. However, low energy heavy ion irradiation may be useful in avoiding the formation of extended defects, but the reactivity between dopant and host 


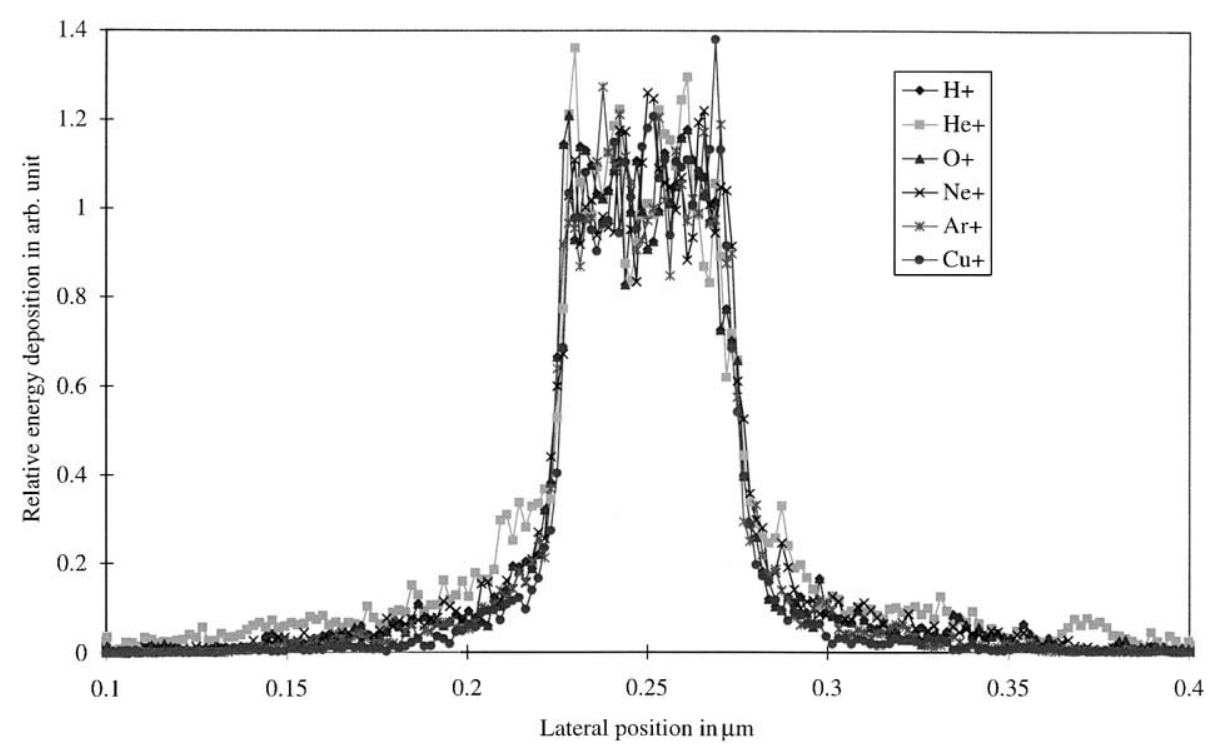

Fig. 2. Scaled irradiation damage distributions along the middle of YBCO thin film covered by a $400 \mathrm{~nm} \mathrm{Nb}$ mask with a $50 \mathrm{~nm}$ opening slot in the centre using different ion species but with $r=2.0$.

ions, oxygen in particular, may damage the definition of well controlled damaged barrier layers, due to the long diffusion distances that are possible. If the diffusion of transition metal ions could be controlled properly, this may provide a new approach for the masked ion damage process.

\section{Conclusions}

The Monte Carlo simulation results described above show that similar irradiation damage profiles can be created by using different ions, provided the beam energies are optimised with the given mask structures. In addition to this, the simulation results also reveal that the irradiation damage broadening effect could be reduced substantially by using the right ion energy so that its range in $\mathrm{Nb}$ is about half of the $\mathrm{Nb}$ mask thickness.

\section{Acknowledgements}

This work is fully supported by EPSRC research grants, GR/L66199, GR/L65963, GR/ M85210, GR/M84756 and GR/L78512.

\section{References}

[1] A.J. Pauza, W.E. Booij, K. Hermann, D.F. Moore, M.G. Blamire, D.A. Rudman, L.R. Vale, Journal of Applied Physics 82 (1997) 5612.

[2] A. Katz, S. Woods, R. Dynes, Journal of Applied Physics 87 (2000) 2978.

[3] W.E. Booij, N.H. Peng, F. Kahlmann, R.P. Webb, E.J. Tarte, D.F. Moore, C. Jeynes, M.G. Blamire, Proceedings of EUCAS 1999, Spain.

[4] F. Kahlmann, A. Engelhardt, J. Schubert, W. Zander, Ch. Buchal, J. Hollkott, Applied Physics Letters 73 (1998) 2032.

[5] I.R. Chakarov, R.P. Webb, Radiation Effects and Defects in Solids 130-133 (1994) 447.

[6] N.H. Peng, I. Chakarov, C. Jeynes, R.P. Webb, W.E. Booij, M.G. Blamire, M.J. Kelly, Nuclear Instruments and Methods in Physics Research B 164-165 (2000) 979985.

[7] N.H. Peng, C. Jeynes, R.P. Webb, I.R. Chakarov, M.G. Blamire, Nuclear Instruments and Methods in Physics Research B 178 (2001) 242-246.

[8] SUSPRE is a program based on energy deposition in the surface and is available from R.P. Webb, SCRIBA, SECM, University of Surrey, UK.

[9] L. Civale, A.D. Marwick, M.W. Mcelfresh, T.K. Worthington, A.P. Malozemoff, F.H. Holzberg, M.A. Kirk, Physical Review Letters 65 (1990) 1164.

[10] J.W. Martin, G.J. Russell, A. Hartmann, D.D. Cohen, Physical Review B 53 (1996) 9412. 